Supporting Materials

\title{
Supramolecular Self-Assembly of Architecturally Variant $\alpha$-Cyclodextrin Inclusion Complexes as Building Blocks of Hexagonally Aligned Microfibrils
}

\author{
Jae Woo Chung, Tae Jin Kang, and Seung-Yeop Kwak ${ }^{*}$
}

School of Materials Science and Engineering, Seoul National University, San 56-1, Sillim-dong, Gwanak-gu, Seoul 151-744, Korea 

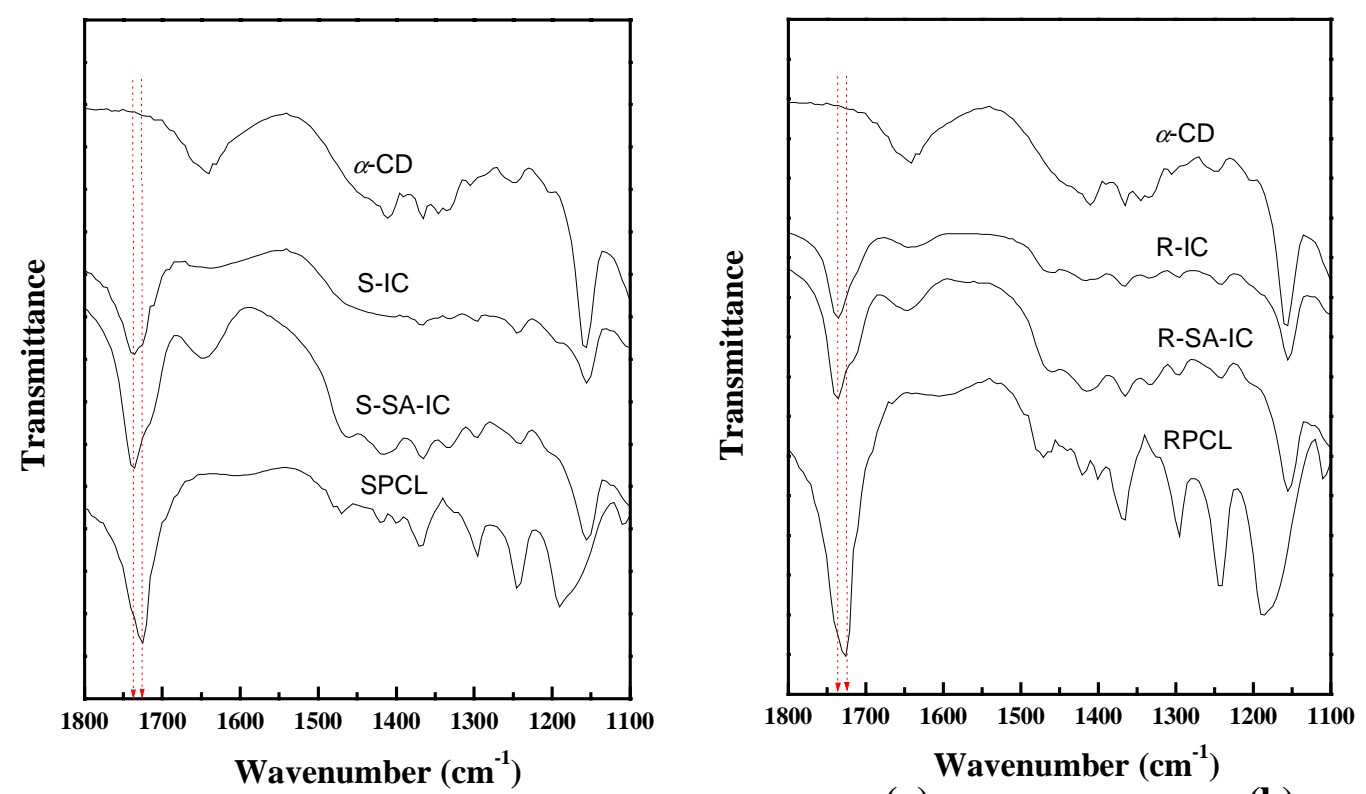

(a)

(b)
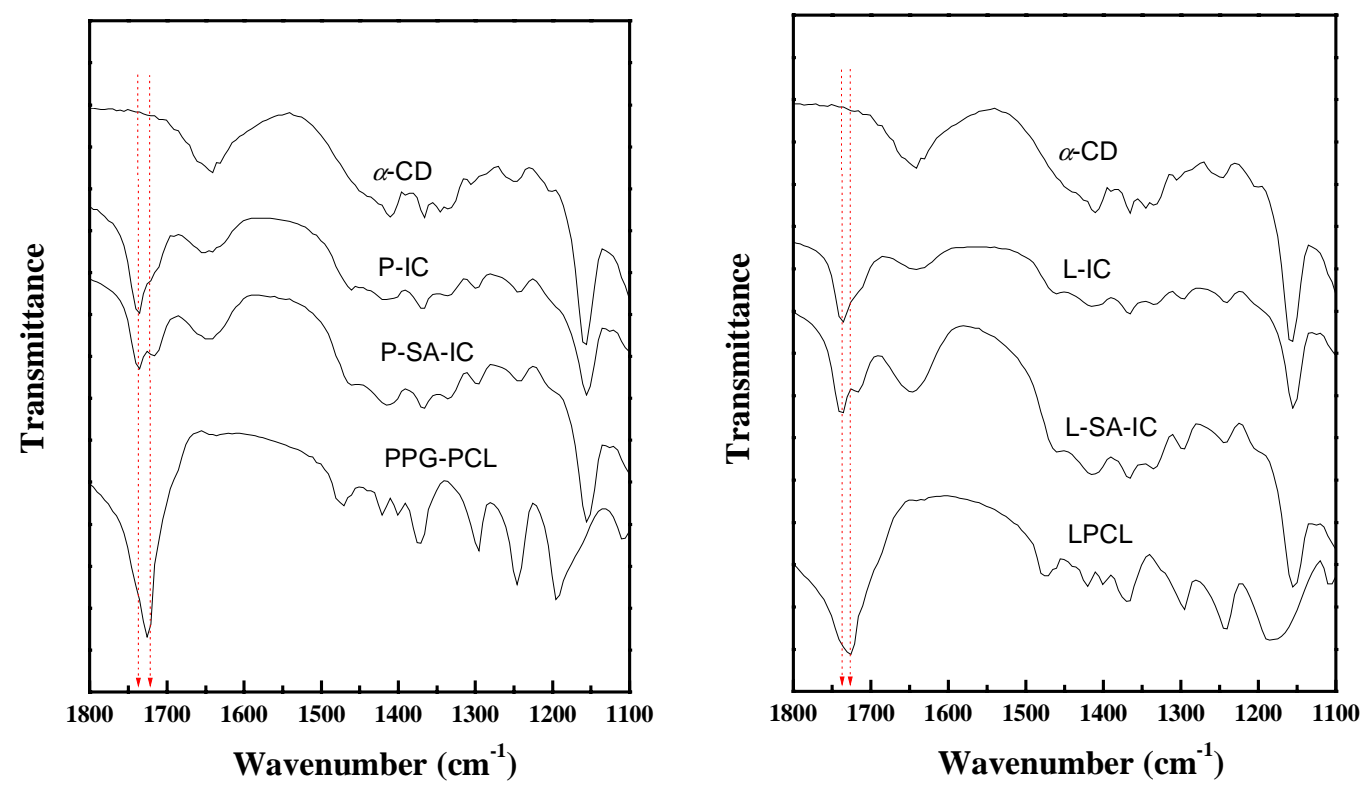

(c)

(d)

Figure S-1. FT-IR spectra of ICs and SA-ICs in which (a) SPCL, (b) RPCL, (c) PPCL, and (d) LPCL was included. 


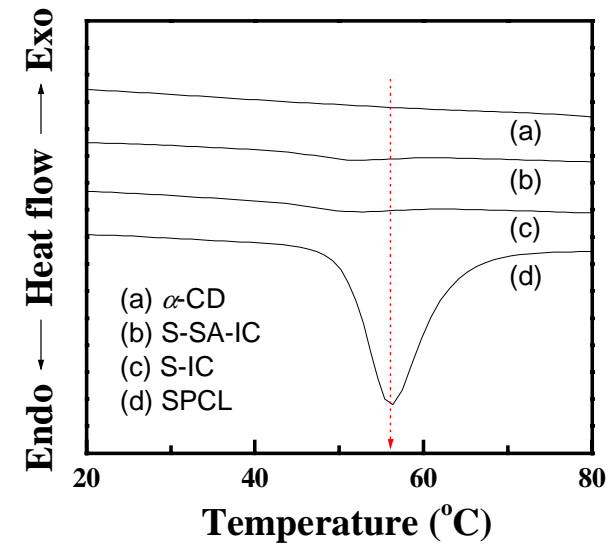

(a)

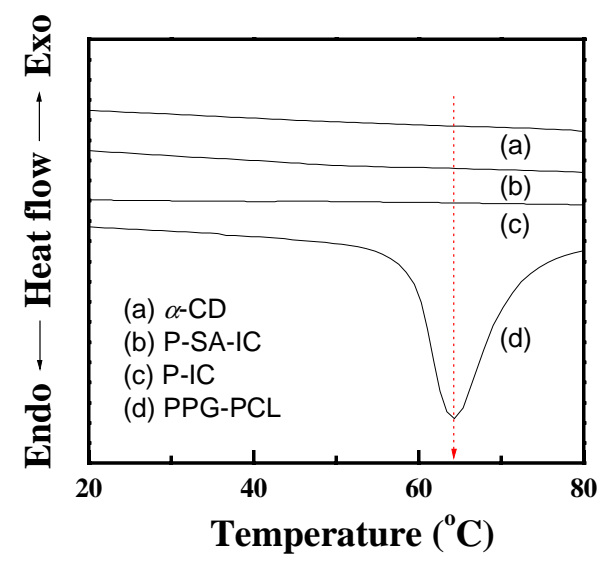

(c)

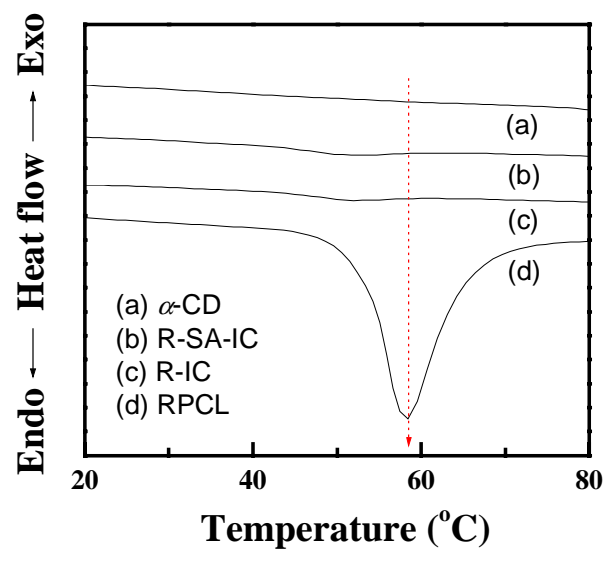

(b)

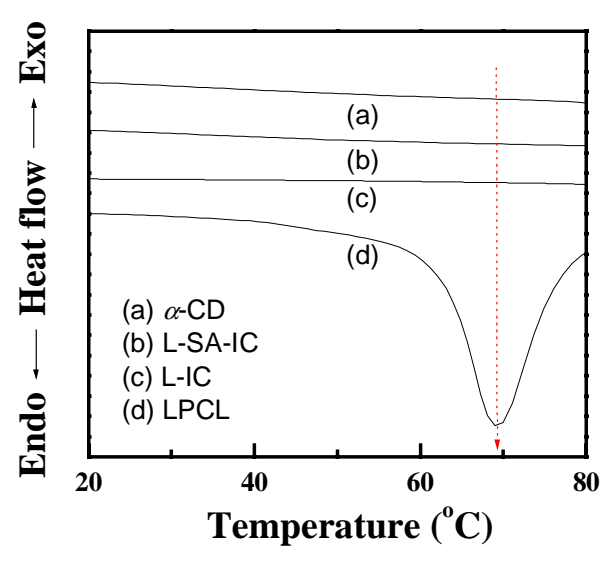

(d)

Figure S-2. DSC curves of ICs and SA-ICs in which (a) SPCL, (b) RPCL, (c) PPCL, and (d) LPCL was included. 


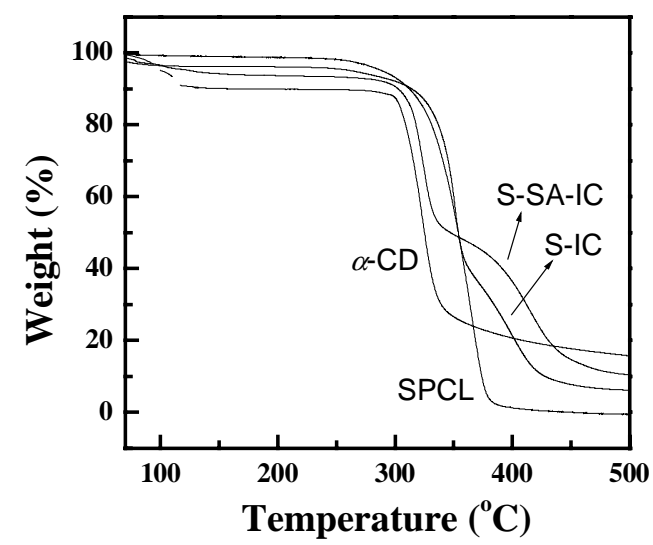

(a)

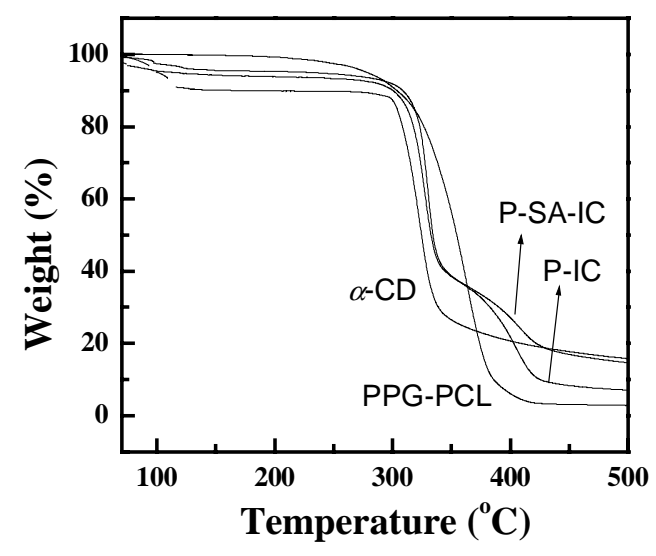

(c)

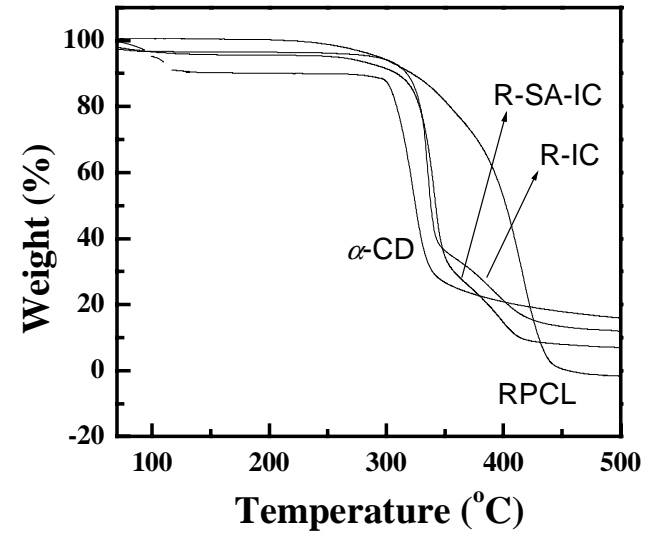

(b)

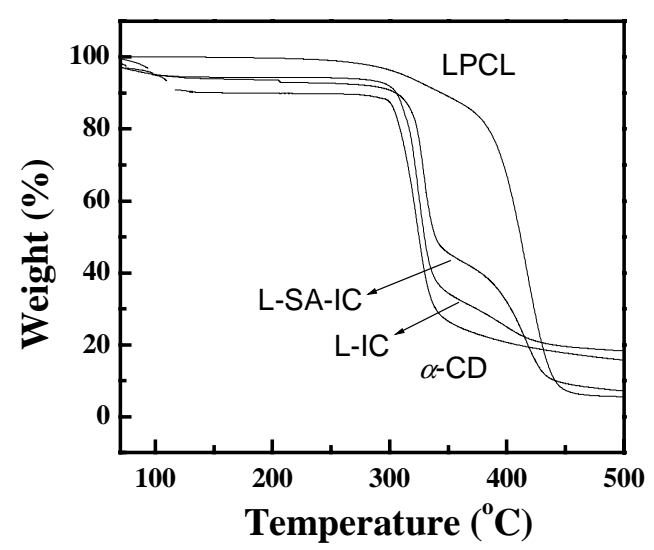

(d)

Figure S-3. TGA scans of ICs and SA-ICs in which (a) SPCL, (b) RPCL, (c) PPCL, and (d) LPCL was included. 

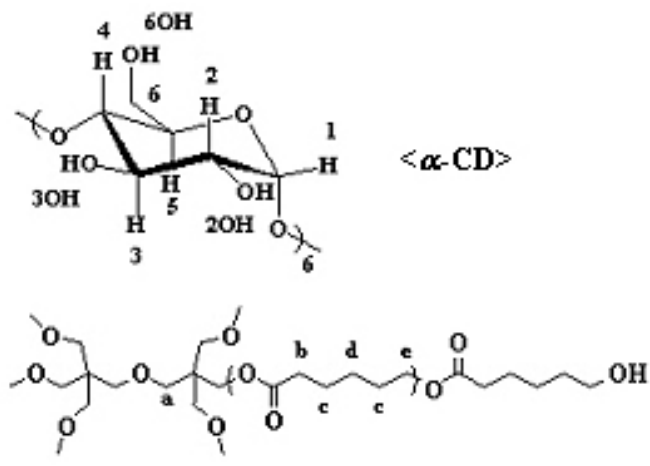

$<$ SPCL $>$

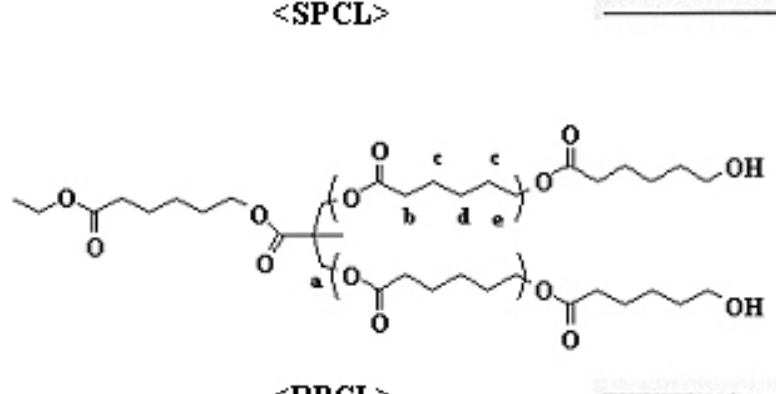

$\langle$ RPCL $>$
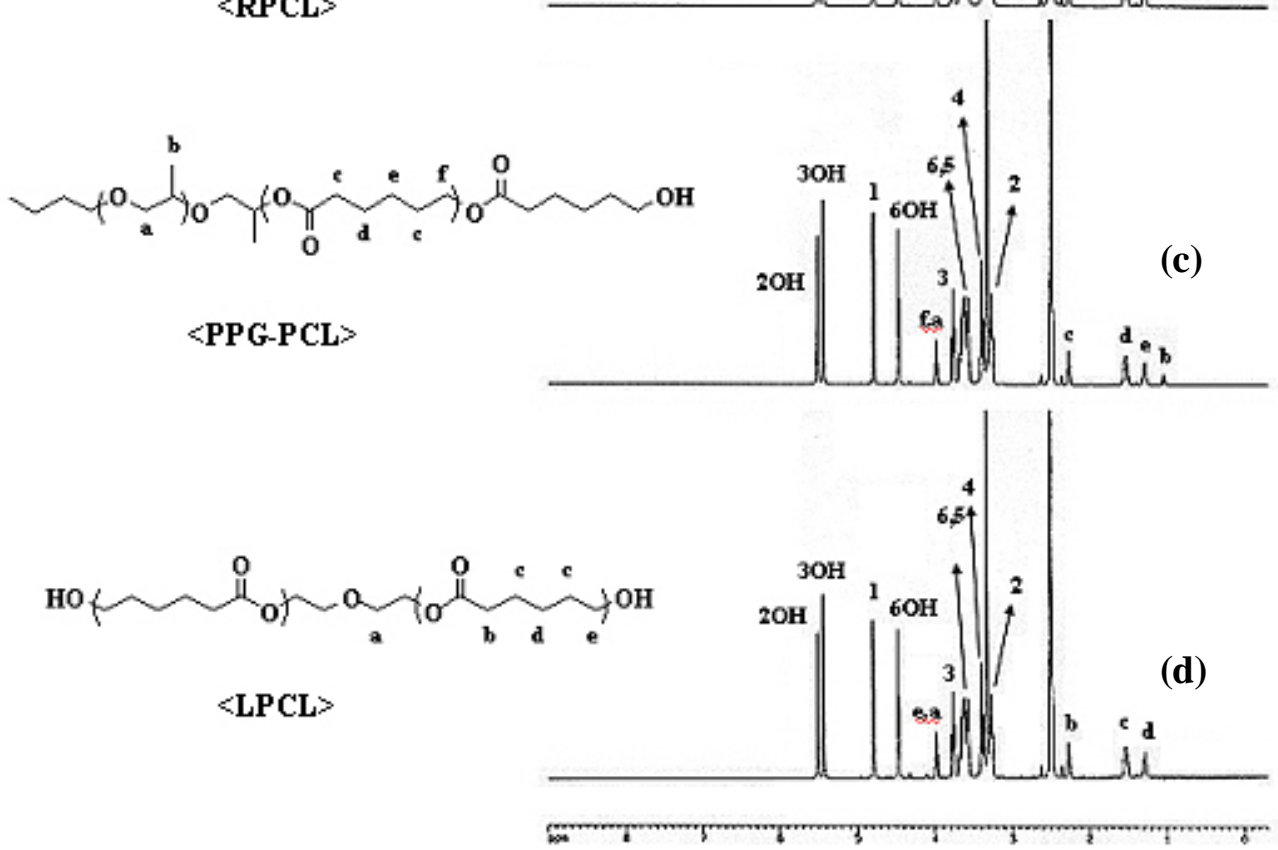

Figure S-4. ${ }^{1} \mathrm{H}$ NMR spectra (DMSO- $d_{6}$ ) of SA-ICs: (a) S-SA-IC, (b) R-SA-IC, (c) PSA-IC, and (d) L-SA-IC. 


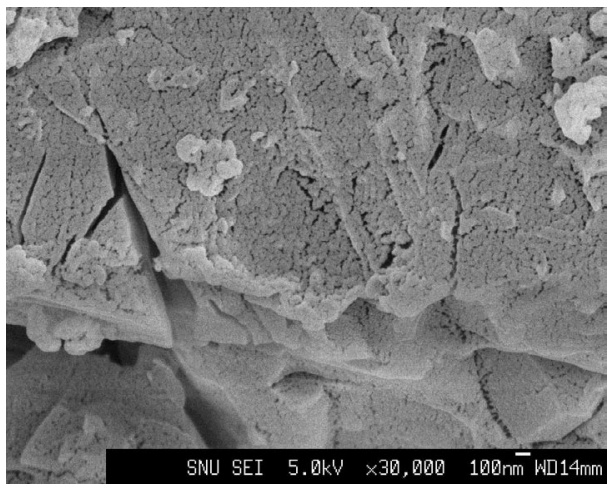

(a)

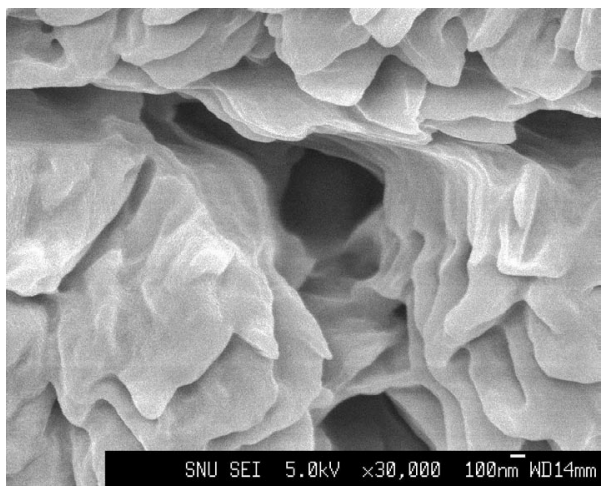

(c)

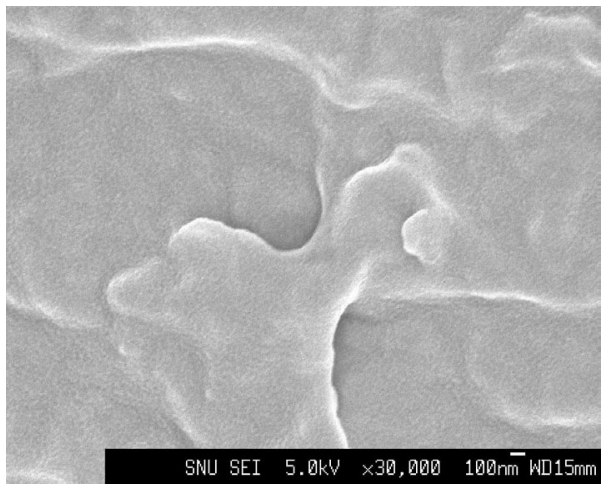

(e)

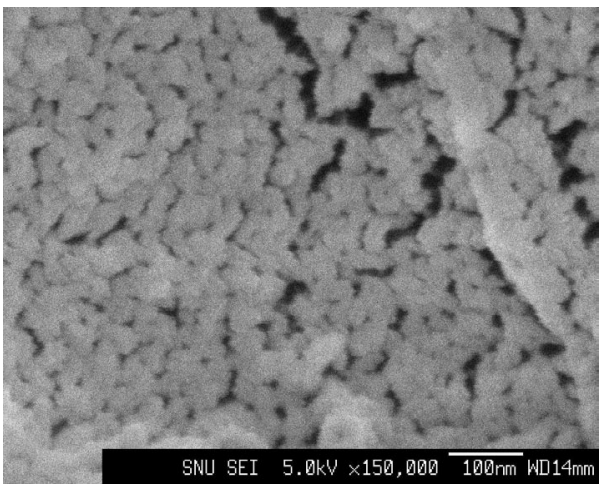

(b)

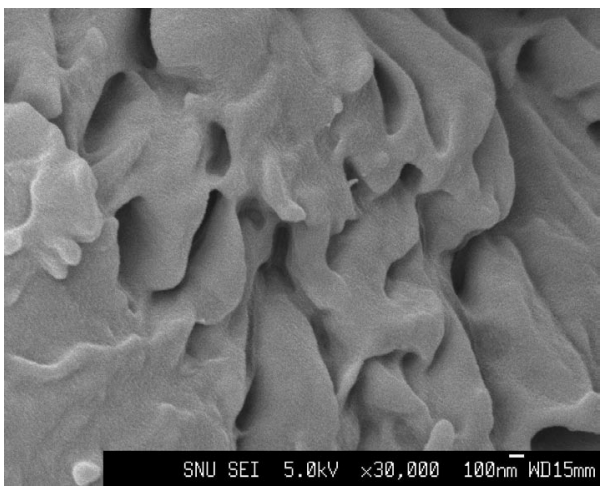

(d)

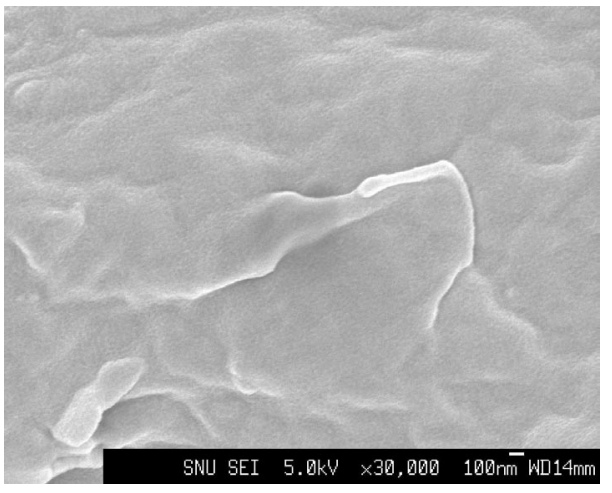

(f)

Figure S-5. FE-SEM images of $\alpha$-CD and PCLs: (a) $\alpha$-CD $(\times 30,000)$, (b) $\alpha$-CD $(\times 150,000)$, (c) SPCL, (d) RPCL, (e) PPG-PCL, and (f) LPCL $(\times 30,000)$. 